\title{
QCD string in excited heavy-light mesons and heavy-quark hybrids
}

\author{
Yu.S. Kalashnikova ${ }^{1,2}$ and A.V. Nefediev ${ }^{1,2,3}$ \\ ${ }^{1}$ Institute for Theoretical and Experimental Physics, \\ 117218, B.Cheremushkinskaya 25, Moscow, Russia \\ ${ }^{2}$ National Research Nuclear University MEPhI, 115409, Kashirskoe highway 31, Moscow, Russia \\ ${ }^{3}$ Moscow Institute of Physics and Technology, 141700, \\ Institutsky lane 9, Dolgoprudny, Moscow Region, Russia
}

\begin{abstract}
The QCD string model is employed to evaluate the masses of orbitally and radially excited heavylight mesons and lightest hybrids in the spectrum of charmonium and bottomonium. The number of parameters of the model is reduced to only seven which are the string tension, the two values of the strong coupling constant (one for heavy-light and $\bar{c} c$ mesons and one for $\bar{b} b$ mesons), and the four overall spectrum shift constants which depend on the quark contents of the particular meson or hybrid family. A few well-established states in the spectrum of heavy-light and heavy-heavy mesons are used to fix these parameters, and then the masses of other mesons and hybrids come out as predictions of the model which are confronted with the existing experimental data, and a few suggestions are made concerning yet not measured quantum numbers of some states in the spectrum of charmonium and bottomonium.
\end{abstract}

PACS numbers: 11.30.Rd, 12.38.Aw, 14.40.-n

\section{INTRODUCTION}

In the last decade, hadronic physics of heavy flavours has experienced a renaissance due to numerous discoveries made in various experiments. In particular, $B$ factories at $e^{+} e^{-}$colliders and the LHC play an especially important role in this process. While $B$-factories typically operate at the energies around the $\Upsilon(4 S)$ bottomonium, they have a potential to scan the region of higher energies, too. Specifically, studies around the $\Upsilon(10860)$ resonance, which is conventionally identified as the $\Upsilon(5 S)$ bottomonium, revealed many new and intriguing features - see, for example, reviews [1 3]. Indeed, at the energies around $11 \mathrm{GeV}$, a few new bottom thresholds are open. For example, studies in the vicinities of the thresholds $B^{(*)} \bar{B}^{*}$ allowed the Belle Collaboration to discover the charged $Z_{b}$ bottomoniumlike resonances [4], which now attract a lot of attention due to their exotic nature. It still remains an open question whether or not the region near the next vector bottomonium, $\Upsilon(11020$, can also be reached for systematic studies by Belle-II but, in any case, additional theoretical information about this region is of paramount importance for the field. For example, an unambiguous identification of the nature of the $\Upsilon(11020)$ resonance and establishing the exact position of the higher-lying open-bottom thresholds are important tasks for future experiments, especially for the $B$-factories of the new generation, like Belle-II. In particular, this amounts to making reliable predictions for the masses of excited heavy-light $B$ mesons as well as for bottomonium hybrids. Meanwhile, the current situation with the spectroscopy of these states looks somewhat ambiguous. From the theory side, in the spectrum of heavy-light mesons containing a heavy quark $Q$, there should exist a positive-parity quadruplet of states $\left(0^{+}, 1^{+}, 1^{+}, 2^{+}\right)$which in the quark-model language corresponds to $P$-level quarkonia. The heavy-quark sym- metry (exact in the limit $m_{Q} \rightarrow \infty$ ) implies a particular splitting pattern within this quadruplet and leads to the formation of two doublets, $\left(0^{+}, 1^{+}\right)$and $\left(1^{+}, 2^{+}\right)$, with a fixed value of the light-quark total momentum, $j_{q}=1 / 2$ and $j_{q}=3 / 2$, respectively. Mass degeneracy within each doublet, exact in the limit $m_{Q} \rightarrow \infty$, is removed for a finite heavy-quark mass, so that the actual splitting pattern between the $P$-level heavy-light mesons may differ substantially from that in the strict heavy-quark limit. All members of the quadruplet in the spectrum of $D$ mesons are known experimentally (see Table I), while the situation with similar states in the spectrum of $B$ mesons is more uncertain since only two states of four are unambiguously identified, and there exists a candidate for the third state (see Table II). In addition, a few more candidate states in the spectrum of $D$ and $B$ mesons exist - see Refs. [5-7] - the quantum numbers of which are not yet identified. Identification of these states and predictions for not yet observed ones is a challenge for phenomenologists.

Another intriguing prediction of QCD is the existence of mesons with an excited gluonic degree of freedomthe so-called hybrids. So far, there is no clear experimental signal of the existence of hybrid mesons; however candidates do appear from time to time. For example, the state $Y(4260)$ [8] demonstrates some feature expected from a charmonium hybrid; namely, it has the mass close to the lattice predictions for such a hybrid [9], and, what is more important, it has a decay pattern (small electronic width and not seen open-charm decays of a particular type) that is not typical for conventional mesons but is specific for hybrids [10 15. However, further studies of the open-charm decays of this state [16] do not confirm its hybrid nature. Discussion of alternative models for $Y(4260)$ can be found in Ref. [17.

There exists a vast literature on hybrids, so let us mention some of many relevant references. For example, re- 
sults of lattice simulations are reported in Refs. [9, [18 23 , and predictions of various models can be found in Refs. 24, 25] (bag model), Ref. [26] (flux-tube model), Refs. 27 29 (Coulomb-gauge QCD approach), Ref. 30 (potential quark model), Refs. [10, 11, 31] (constituent gluon model), and Refs. 32 38. (QCD string approach).

In this paper, the QCD string approach is used to provide a self-consistent description of heavy-light radially and orbitally excited $D$ and $B$ mesons, low-lying heavy $\bar{c} c$ and $\bar{b} b$ mesons, and the lowest $\bar{c} c g$ and $\bar{b} b g$ hybrids. Parameters of the corresponding Hamiltonians are totally fixed from the masses of a few well-established heavylight and heavy-heavy mesons. Then, the masses of other heavy-light mesons, including radially excited as well as $P$ - and $D$-wave ones, come out as predictions. Also, in the given approach, the lowest vector bottomonium hybrid is predicted to possess the mass around $11.04 \mathrm{GeV}$ that places it just in the vicinity of the $B^{(*)} B_{J}$ thresholds, with $B_{J}(J=0,1,2)$ denoting the quadruplet of positive-parity $B$-mesons. Constraints from the heavyquark spin symmetry which suppress decays for a genuine vector bottomonium to the corresponding open-bottom channels 39 may give us a clue to understanding the nature of the $\Upsilon(11020)$ resonance. The results obtained emphasise the importance of studies of the energy region around $11 \mathrm{GeV}$ in the future high-statistics and highprecision experiments and, in particular, are expected to be relevant for the physical programme of the nextgeneration $B$-factories.

\section{HAMILTONIANS OF MESONS AND HYBRIDS}

The QCD string model has a long history. It is based on the Vacuum Background Correlators Method (see review 40, and references therein), and its application to the simplest hadronic system-the quark-antiquark meson - can be found in Refs. 41, 42. A complementary approach which radically simplifies the algebra related to the relativistic kinematics is the einbein field formalism 43. It allows one to reduce the fully relativistic kinematics to an effectively nonrelativistic one with the help of auxiliary degrees of freedom provided by the einbeins. The details of the formalism and relevant references can be found in Ref. 44. If einbeins are treated as variational parameters, the suggested approach is applicable to a wide class of hadronic systems, including hybrids and glueballs [33, 41, 45, 46]. A detailed discussion of the variational procedure based on the einebin field approach can be found in Ref. 47.

In the QCD string approach, the Hamiltonian of a hadron can be written in the form

$$
H=H_{0}+V_{\mathrm{str}}+V_{\mathrm{SD}},
$$

where $H_{0}$ describes the dynamics of the spinless quarks interacting through the linear-plus-Coulomb potential, $V_{\text {str }}$ is the string correction which accounts for the proper dynamics of the QCD string [41, and $V_{\mathrm{SD}}$ describes spindependent interactions. In particular, for the quark-antiquark meson in its centre-of-mass frame one has [47 51 .

$$
\begin{gathered}
H_{0}=\sum_{i=1}^{2}\left(\frac{\boldsymbol{p}^{2}+m_{i}^{2}}{2 \mu_{i}}+\frac{\mu_{i}}{2}\right)+\sigma r+V_{\mathrm{Coul}}-C_{0}, \\
V_{\mathrm{Coul}}=-\frac{4}{3} \frac{\alpha_{S}}{r}, \quad V_{\mathrm{str}}=-\frac{\sigma\left(\mu_{1}^{2}+\mu_{2}^{2}-\mu_{1} \mu_{2}\right)}{6 \mu_{1}^{2} \mu_{2}^{2}} \frac{\boldsymbol{L}^{2}}{r}, \\
V_{\mathrm{SD}}=V_{\mathrm{LS}}+V_{\mathrm{SS}}+V_{\mathrm{ST}},
\end{gathered}
$$

where the subscripts LS, SS, and ST stand for the spinorbital, hyperfine, and spin-tensor interaction, respectively (for the details see, for example, Ref. 50 ). The constant $C_{0}$ provides an overall shift of the spectrum. The quantities $\mu_{1,2}$ are the einbein fields interpreted as dynamical masses of the quarks. For each particular eigenstate of Hamiltonian (1) their values are found from the requirement that the corresponding eigenenergy takes an extremal value.

Similarly, for a hybrid meson containing two quarks and a gluon one has [36, 52,

$$
\begin{gathered}
H_{0}=\frac{\mu_{q}+\mu_{\bar{q}}+\mu_{g}}{2}+\frac{m_{q}^{2}+\boldsymbol{p}_{q}^{2}}{2 \mu_{q}}+\frac{m_{\bar{q}}^{2}+\boldsymbol{p}_{\bar{q}}^{2}}{2 \mu_{\bar{q}}}+\frac{\boldsymbol{p}_{g}^{2}}{2 \mu_{g}} \\
+\sigma\left|\boldsymbol{r}_{q}-\boldsymbol{r}_{g}\right|+\sigma\left|\boldsymbol{r}_{\bar{q}}-\boldsymbol{r}_{g}\right|+V_{\mathrm{Coul}}-C_{0}, \\
V_{\mathrm{Coul}}=-\frac{3 \alpha_{s}}{2\left|\boldsymbol{r}_{q}-\boldsymbol{r}_{g}\right|}-\frac{3 \alpha_{s}}{2\left|\boldsymbol{r}_{\bar{q}}-\boldsymbol{r}_{g}\right|}+\frac{\alpha_{s}}{6\left|\boldsymbol{r}_{q}-\boldsymbol{r}_{\bar{q}}\right|}, \\
V_{\mathrm{SD}}=V_{\mathrm{LS}}^{(q \bar{q})}+V_{\mathrm{LS}}^{(g)}+V_{\mathrm{SS}}+V_{\mathrm{ST}}^{(q \bar{q})}+V_{\mathrm{ST}}^{(g)},
\end{gathered}
$$

where $V_{\text {Coul }}$ describes the pairwise colour Coulomb interactions [31, and the string correction (not quoted here) depends on the angular momenta between the quarks and the gluon. For hybrids with the quark and the antiquark of the same flavour one can set $m_{q}=m_{\bar{q}}=m$, so that $\mu_{q}=\mu_{\bar{q}}=\mu$, and the centre-of-mass motion in this three-body system can be separated with the help of the standard Jacobi coordinates,

$$
\boldsymbol{r}=\boldsymbol{r}_{q}-\boldsymbol{r}_{\bar{q}}, \quad \boldsymbol{\rho}=\boldsymbol{r}_{g}-\frac{\mu_{q} \boldsymbol{r}_{q}+\mu_{\bar{q}} \boldsymbol{r}_{\bar{q}}}{\mu_{q}+\mu_{\bar{q}}}=\boldsymbol{r}_{g}-\frac{\boldsymbol{r}_{q}+\boldsymbol{r}_{\bar{q}}}{2},
$$

defined in terms of the effective dynamical masses of the quarks. For the explicit form of the Hamiltonian used in the calculations and for further details see Ref. 36.

Due to the presence of extra degrees of freedom, hybrids possess properties severely different from the properties of conventional quark-antiquark mesons. In particular, while quantum numbers of the latter follow the standard scheme, $P=(-1)^{l_{q \bar{q}}+1}$ and $C=(-1)^{l_{q \bar{q}}+s_{q \bar{q}}}$, so that exotic quantum numbers $1^{-+}$are not accessible, for the one-gluon hybrid one can find that (see Ref. [36] and references therein)

$$
P=(-1)^{l_{q \bar{q}}+j}, \quad C=(-1)^{l_{q \bar{q}}+s_{q \bar{q}}+1},
$$


for the magnetic hybrid $\left(l_{g}=j\right)$ and

$$
P=(-1)^{l_{q \bar{q}}+j+1}, \quad C=(-1)^{l_{q \bar{q}}+s_{q \bar{q}}+1},
$$

for the electric hybrid $\left(l_{g}=j \pm 1\right)$, where $l_{g}$ is the angular momentum of the gluon relative to the quark-antiquark pair, $j$ is the total momentum of the gluon, and $l_{q \bar{q}}, s_{q \bar{q}}$ are the angular momentum and the spin of the quarkantiquark system, respectively. So, the given quantum numbers can be achieved both for electric and magnetic hybrids.

Notice that electric hybrids possess such a large decay width into two $S$-wave heavy-light mesons that they can hardly be observed [11. The situation for the magnetic hybrid is opposite because such a decay is forbidden for it by a well-known selection rule [10 15. Then, while decays into one $S$-wave and one $P$-wave meson with open flavour are allowed, the corresponding widths are relatively small. Thus, in what follows, only lowest magnetic hybrids will be considered, namely the vector $1^{--}$one with

$$
s_{q \bar{q}}=0, \quad l_{q \bar{q}}=0, \quad l_{g}=1, \quad j=1
$$

and three $C$-even $J^{-+}(J=0,1,2)$ siblings with

$$
s_{q \bar{q}}=1, \quad l_{q \bar{q}}=0, \quad l_{g}=1, \quad j=1 .
$$

\section{PARAMETERS AND PROCEDURE}

The standard procedure to deal with Hamiltonian (1) is to solve the corresponding Schödinger equation for the leading-order term $H_{0}$ and then to include other terms as perturbations. Further details can be found in Refs. 36, 47, 49 51. It should be noticed that, unlike previous works, in this paper the number of parameters of the model is reduced to a minimum; in particular, the quark masses are not treated as free parameters, and the remaining seven parameters are fixed in a self-consistent way for all hadronic systems discussed. Also, updated experimental data are used. Thus, in what follows, the masses of the quarks take their standard pole values evaluated in two loops [5]. Since the isospin effects lie beyond the scope of this research, then for the light quark $q$ the averaged value between the $u$ quark mass and the $d$ quark mass is used. Therefore,

$$
m_{q}=3.6 \mathrm{MeV}, m_{c}=1.67 \mathrm{GeV}, m_{b}=4.78 \mathrm{GeV}
$$

Then the set of parameters of the model is given by the string tension $\sigma$, the strong coupling constant $\alpha_{s}$, and the overall constant shift of the spectrum $C_{0}$. The parameters $\sigma$ and $\alpha_{S}$ can be somewhat adapted to a particular system under study; however, they are strongly constrained by phenomenology. In particular, the string tension takes its standard value consistent with phenomenology - see Table VII. The situation with the strong coupling constant is somewhat more subtle. It demonstrates a dependence on the scale which can be presented as 53 .

$$
\alpha_{S}\left(Q^{2}\right)=\left(b_{0} \ln \frac{Q^{2}+\mathcal{M}^{2}}{\Lambda_{\mathrm{QCD}}^{2}}\right)^{-1},
$$

where $b_{0}$ is the one-loop coefficient of the $\beta$-function, $\Lambda_{\mathrm{QCD}}$ is the standard parameter of $\mathrm{QCD}$, and $\mathcal{M}$ takes values of the order of $1-2 \mathrm{GeV}$ - see the discussion and relevant references in Ref. [53. It is easy to see that $\mathcal{M} \simeq m_{c}$, so that, for the scales below $m_{c}, \alpha_{S}$ remains nearly constant and takes values close to the "frozen" limit $\alpha_{S}^{\mathrm{fr}} \approx 0.6$. Meanwhile, since $m_{b} \simeq(3-4) \mathcal{M}$, then it is natural to expect a smaller $\alpha_{s}$ in the $\bar{b} b$ bottomonia. In other words, the following hierarchy of the values of $\alpha_{S}$ is expected,

$$
\alpha_{S}\left(m_{q}\right) \approx \alpha_{S}\left(m_{c}\right)>\alpha_{S}\left(m_{b}\right)
$$

that implies that $(Q=c, b)$

$$
\alpha_{S}^{\bar{Q} q} \approx \alpha_{S}^{\bar{c} c} \simeq 0.5-0.6, \quad \alpha_{S}^{\bar{b} b} \simeq 0.4-0.5 .
$$

The constant $C_{0}$ is treated as a free parameter of the model, and we take it to be the same in both quarkonium and hybrid sectors. As shown in Ref. [54, this constant can be viewed as the quark self-energy which takes into account the bare quark mass renormalisation due to the confining background. Obviously, such a renormalisation is absent for gluons because of gauge invariance. Following this reasoning we also assume that the constant $C_{0}$ appears as the quark self-energy while the gluon self-energy vanishes. This assumption finds a further phenomenological justification in the calculations of the glueball spectrum in the QCD string approach [55]: the calculated glueball masses, with the gluon self-energy set equal to zero, agree well with the masses found on the lattice.

Therefore, the following procedure is adopted. First, the model is fully fixed and verified as follows:

- The spectrum of the $P$-level $D$ mesons (four states) is calculated - see Table $\mathrm{I}$-and parameters $\sigma, \alpha_{S}^{\bar{c} q}$, and $C_{0}^{\bar{c} q}$ are adjusted to provide the best overall description of the experimentally observed masses. If both neutral $\left(m_{0}\right)$ and charged $\left(m_{ \pm}\right)$mesons are measured, the isospin averaged value $\left(2 m_{ \pm}+m_{0}\right) / 3$ is used in the fit.

- The masses of the well-established $P$-level $B$ mesons (two states) are calculated with the string tension and the coupling $\alpha_{S}$ taking the values found above, from the fit for the $D$-meson masses - see Table II and the only free parameter, the constant $C_{0}^{b q}$, is adjusted this way. Notice that, since $C_{0}$ only provides the overall shift of the spectrum, then the splittings between the $B$ mesons are predictions. 


\begin{tabular}{|c|c|c|c|c|c|c|}
\hline${ }^{2 S+1} L_{J}(\mathrm{HQ}$ term $)$ & Meson & $J^{P}$ & Mass (theor. $), \mathrm{MeV}$ & Width (theor.) & Mass (exp. $), \mathrm{MeV}$ & Width (exp.), MeV \\
\hline${ }^{3} P_{0}\left(P_{1 / 2}\right)$ & $D_{0}(2400)$ & $0^{+}$ & 2343 & broad & $2318 \pm 29 / 2403 \pm 40$ & $267 \pm 40 / 283 \pm 40$ \\
\hline$P_{1}^{l}\left(P_{1 / 2} \cos \theta_{D}-P_{3 / 2} \sin \theta_{D}\right)$ & $D_{1}(2420)$ & $1^{+}$ & 2423 & narrow & $2421.4 \pm 0.6 / 2423.2 \pm 2.4$ & $27.4 \pm 2.5 / 25 \pm 6$ \\
\hline$P_{1}^{h}\left(P_{1 / 2} \sin \theta_{D}+P_{3 / 2} \cos \theta_{D}\right)$ & $D_{1}(2430)$ & $1^{+}$ & 2441 & broad & $2427 \pm 40 /-$ & $384_{-110}^{+130} /-$ \\
\hline${ }^{3} P_{2}\left(P_{3 / 2}\right)$ & $D_{2}(2460)$ & $2^{+}$ & 2463 & narrow & $2462.6 \pm 0.6 / 2464.3 \pm 1.6$ & $49.0 \pm 1.3 / 37 \pm 6$ \\
\hline
\end{tabular}

TABLE I. Masses of $P$-level $D$ mesons. $P_{1 / 2}$ and $P_{3 / 2}$ indicate the heavy-quark (HQ) states doubly degenerate in the strict limit $m_{c} \rightarrow \infty$. The mixing angle is $\theta_{D} \approx 60^{\circ}$. Experimental data are taken from the live update of PDG [5] and are quoted as $M\left(D_{J}^{0}\right) / M\left(D_{J}^{ \pm}\right)$.

\begin{tabular}{|c|c|c|c|c|c|c|}
\hline${ }^{2 S+1} L_{J}(\mathrm{HQ}$ term $)$ & Meson & $J^{P}$ & Mass (theor.), MeV & Width (theor.) & Mass (exp.), MeV & Width (exp.), MeV \\
\hline${ }^{3} P_{0}\left(P_{1 / 2}\right)$ & $B_{J}^{*}(5732)$ & $0^{+}$ & 5669 & broad & $5698 \pm 8(?)$ & $128 \pm 18(?)$ \\
\hline$P_{1}^{l}\left(P_{1 / 2} \cos \theta_{B}-P_{3 / 2} \sin \theta_{B}\right)$ & $B_{1}$ & $1^{+}$ & 5713 & broad & - & - \\
\hline$P_{1}^{h}\left(P_{1 / 2} \sin \theta_{B}+P_{3 / 2} \cos \theta_{B}\right)$ & $B_{1}(5721)$ & $1^{+}$ & 5724 & narrow & $5724.9 \pm 2.4 / 5726.8_{-4.0}^{+3.2}$ & $23 \pm 5 / 49_{-16}^{+12}$ \\
\hline${ }^{3} P_{2}\left(P_{3 / 2}\right)$ & $B_{2}^{*}(5747)$ & $2^{+}$ & 5741 & narrow & $5739 \pm 5 / 5736.9_{-1.6}^{+1.3}$ & $22 \pm 5 / 11 \pm 5$ \\
\hline
\end{tabular}

TABLE II. Masses of $P$-level $B$ mesons. $P_{1 / 2}$ and $P_{3 / 2}$ indicate the heavy-quark (HQ) states doubly degenerate in the strict limit $m_{b} \rightarrow \infty$. The mixing angle is $\theta_{B} \approx 24^{\circ}$. Experimental data are taken from the live update of PDG [5] and are quoted as $M\left(B_{J}^{0}\right) / M\left(B_{J}^{ \pm}\right)$. State $B_{J}^{*}(5732)$, not yet confirmed and therefore tagged with the question mark, is placed in the most appropriate cell according to its mass and width quoted in PDG [5].

- The spectrum of the low-lying $\bar{c} c$ mesons (six states) is calculated - see Table $\mathrm{V}$ - and the constant $C_{0}^{\bar{c} c}$ is fixed this way. As before, the splittings between the levels are not adjusted and come as predictions.

- The spectrum of the low-lying $\bar{b} b$ mesons (six states) is calculated - see Table $\mathrm{VI}$ and the only remaining free parameters of the model, $\alpha_{S}^{\bar{b} b}$ and $C_{0}^{\bar{b} b}$, are determined.

For convenience, the values of the parameters extracted as explained above are collected in Table VII. It is worthwhile noticing that the values of $\alpha_{S}$ comply quite well with relation (13) and, in particular, fall into the ranges quoted in Eq. (14). This provides an additional self-consistence test for the approach.

For completeness, we quote here the values of the auxiliary parameters $\mu_{1}$ and $\mu_{2}$ as they come out from the calculations,

$$
\begin{aligned}
& \mu_{1}(\bar{c} q ; 1 P)=1781 \mathrm{MeV}, \mu_{2}(\bar{c} q ; 1 P)=618 \mathrm{MeV} \\
& \mu_{1}(\bar{b} q ; 1 P)=4830 \mathrm{MeV}, \mu_{2}(\bar{b} q ; 1 P)=694 \mathrm{MeV} \\
& \mu_{1}(\bar{b} q ; 1 D)=4840 \mathrm{MeV}, \mu_{2}(\bar{b} q ; 1 D)=765 \mathrm{MeV} \\
& \mu_{1}(\bar{b} q ; 2 S)=4847 \mathrm{MeV}, \mu_{2}(\bar{b} q ; 2 S)=801 \mathrm{MeV}
\end{aligned}
$$

where in parentheses we give the quark contents of the heavy-light system and its quantum numbers.

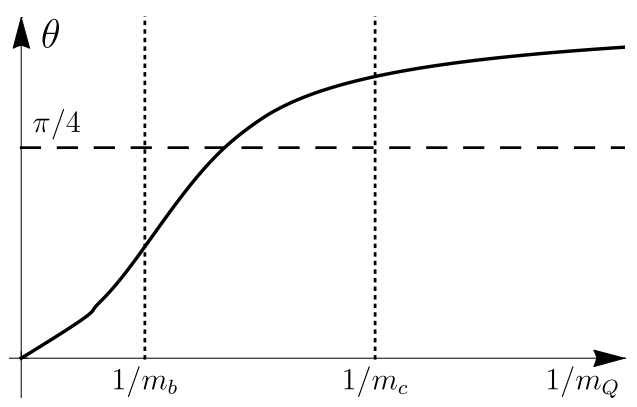

FIG. 1. The mixing angle $\theta$ for the $P$ levels as a function of the inversed heavy-quark mass. The physical points for the $b$ and $c$ quarks are shown with vertical dotted lines.

\section{RESULTS FOR HEAVY-LIGHT MESONS}

Now, with the details of the approach described in the previous section and with the complete set of parameters of the model fixed as quoted in Eq. (11) and in Table VII. we are in a position to turn to various predictions of the model. We start from the heavy-light $D$ and $B$ mesonssee Tables [and[I]. As was explained above, the masses of the six well-established experimentally states were used as input to fix the parameters of the model. From Table I. one can see that the model is able to describe the spectrum of the $P$-wave $D$ mesons with a sufficiently high accuracy. The same conclusion holds for the two known positive-parity $B$ mesons.

It has to be noticed that, in order to proceed with the 


\begin{tabular}{|c|c|c|c|c|c|c|}
\hline Term & $2^{1} S_{0}$ & $2^{3} S_{1}$ & $1^{3} D_{3}$ & $1 D_{2}^{l}$ & $1 D_{2}^{h}$ & $1^{3} D_{1}$ \\
\hline$J^{P}$ & $0^{-}$ & $1^{-}$ & $3^{-}$ & $2^{-}$ & $2^{-}$ & $1^{-}$ \\
\hline Mass (theor.), MeV & 2532 & 2697 & 2682 & 2693 & 2794 & 2811 \\
\hline Mass (exp.), MeV & $2539 \pm 8$ & $2612 \pm 6$ & $2637 \pm 6$ & - & $2761 \pm 5$ & - \\
\hline Hypothesis $\mathcal{D}_{1}(51)$ & $D(2550)$ & $D(2600)$ & $D(2640)$ & - & $D(2750)$ & - \\
\hline Hypothesis $\mathcal{D}_{2}(54)$ & $D(2550)$ & $D(2600)$ & $D(2640)$ & - & - & $D(2750)$ \\
\hline
\end{tabular}

TABLE III. Masses of radially and orbitally excited $D$ mesons predicted by the model and their possible identification with experimentally observed states taken from Ref. [5]. The number in parentheses in the hypothesis name gives the mean quadratic deviation (in $\mathrm{MeV}$ ) of the theoretical predictions from the experimental masses.

\begin{tabular}{|c|c|c|c|c|c|c|}
\hline Term & $2^{1} S_{0}$ & $2^{3} S_{1}$ & $1^{3} D_{3}$ & $1 D_{2}^{l}$ & $1 D_{2}^{h}$ & $1^{3} D_{1}$ \\
\hline$J^{P}$ & $0^{-}$ & $1^{-}$ & $1^{-}$ & $2^{-}$ & $2^{-}$ & $3^{-}$ \\
\hline Spin-parity type & $\mathrm{UN}$ & $\mathrm{N}$ & $\mathrm{N}$ & $\mathrm{UN}$ & $\mathrm{UN}$ & $\mathrm{N}$ \\
\hline Mass (theor.), MeV & 5853 & 5942 & 5961 & 5962 & 6061 & 6064 \\
\hline Hypothesis $\mathcal{B}_{1}(80)$ & - & $B(5970)$ & - & $B_{J}(5840)$ & $B_{J}(5960)$ & - \\
\hline Hypothesis $\mathcal{B}_{2}(79)$ & - & - & $B(5970)$ & $B_{J}(5840)$ & $B_{J}(5960)$ & - \\
\hline Hypothesis $\mathcal{B}_{3}(36)$ & $B_{J}(5840)$ & $B(5970)$ & - & - & $B_{J}(5960)$ & - \\
\hline Hypothesis $\mathcal{B}_{4}(32)$ & $B_{J}(5840)$ & - & $B(5970)$ & - & $B_{J}(5960)$ & - \\
\hline Hypothesis $\mathcal{B}_{5}(63)$ & - & $B(5970)$ & - & $B_{J}(5840)$ & - & $B_{J}(5960)$ \\
\hline Hypothesis $\mathcal{B}_{6}(61)$ & - & - & $B(5970)$ & $B_{J}(5840)$ & - & $B_{J}(5960)$ \\
\hline
\end{tabular}

TABLE IV. Masses of radially and orbitally excited $B$ mesons predicted by the model and their possible identification with experimentally observed states taken from Refs. 5 and 7 . The number in parentheses in the hypothesis name gives the mean quadratic deviation (in $\mathrm{MeV}$ ) of the theoretical predictions from the experimental masses. The spin-parity scheme corresponds to the one used in Ref. [7]: natural (N) spin-parity implies that $P=(-1)^{J}$ while unnatural (UN) spin-parity implies that $P=(-1)^{J+1}$.

identification of the heavy-light mesons, it is important to understand the splitting pattern in the $P$-level quadruplet. As was mentioned in the Introduction, the heavyquark symmetry implies the formation of two degenerate doublets, $\left(0^{+}, 1^{+}\right)$and $\left(1^{+}, 2^{+}\right)$, with a fixed value of the light-quark total momentum, $j_{q}=1 / 2$ and $j_{q}=3 / 2$, respectively. Notice also that the total quark spin is not a good quantum number in the system which does not possess $C$-parity, so that the $P$-level states with the same total momentum but with different total spins are mixed with the spin-orbit interaction and the observed mesons appear as particular combinations of the latter. The mixing can be parametrised through the mixing angle $\theta$ as (see Appendix $\mathrm{A}$ for the details)

$$
\left(\begin{array}{c}
P_{1}^{l} \\
P_{1}^{h}
\end{array}\right)=\left(\begin{array}{cc}
\cos \theta & -\sin \theta \\
\sin \theta & \cos \theta
\end{array}\right)\left(\begin{array}{l}
P_{1 / 2} \\
P_{3 / 2}
\end{array}\right),
$$

where the superscript $l(h)$ denotes the light(heavy) member of the doublet.

The dependence of the mixing angle on the heavyquark mass, as predicted by our model, is shown in Fig. 1. It is seen from the figure that the mixing angles for the $D$ and $B$ mesons lie on different sides from the line $\theta=\pi / 4$ that implies that the $P$ levels in the two systems follow each other in a different order. Indeed, in the $D$ mesons, $\theta_{D} \approx 60^{\circ}>45^{\circ}$ and therefore the states which are completely or predominantly given by the $P_{1 / 2}$ and $P_{3 / 2}$ levels follow one by one. Heavy-quark symmetry constraints imply that the $P_{1 / 2}$ states couple to a heavylight ground-state meson and pion in the $S$ wave while the $P_{3 / 2}$ ones couple to a heavy-light ground-state meson and pion in the $D$ wave. Thus, one expects the $P_{1 / 2}$ states to be broad and the $P_{3 / 2}$ states to be narrow, so that the width pattern for the $D$ mesons is predicted by our model to be (broad,narrow,broad,narrow), starting from the lightest state - see Table I. Conversely, for the $B$ mesons, $\theta_{B} \approx 24^{\circ}<45^{\circ}$, so that the width pattern is different, namely (broad,broad,narrow,narrow) - see Table III This makes a crucial difference between the splitting patterns of the $P$-level $D$ and $B$ mesons.

According to this scheme, the two not yet identified members of the positive-parity quadruplet of the $B$ mesons with the quantum numbers $0^{+}$and $1^{+}$are expected to be broad, with the width of the order of a few hundred $\mathrm{MeV}$. Their masses are predicted to take the values around 5700 and $5730 \mathrm{MeV}$, respectively. Then, the observed state $B_{J}^{*}(5732)$ [5], if confirmed, can be identified as the scalar meson $B_{0}$ which, in agreement with the qualitative prediction of the model, is broad - see Table II

As the next step, the masses of several radially $(n=2)$ 


\begin{tabular}{|c|c|c|c|c|c|c|}
\hline Meson & $\eta_{c}(1 S)$ & $J / \psi(1 S)$ & $h_{c}(1 P)$ & $\chi_{c_{1}}(1 P)$ & $\chi_{c_{0}}(1 P)$ & $\chi_{c_{2}}(1 P)$ \\
\hline$J^{P}$ & $0^{-+}$ & $1^{--}$ & $1^{+-}$ & $1^{++}$ & $0^{++}$ & $2^{++}$ \\
\hline${ }^{2 S+1} L_{J}$ & ${ }^{1} S_{0}$ & ${ }^{3} S_{1}$ & ${ }^{1} P_{1}$ & ${ }^{3} P_{1}$ & ${ }^{3} P_{0}$ & ${ }^{3} P_{2}$ \\
\hline Exp., MeV [5] & 2984 & 3097 & 3525 & 3511 & 3415 & 3556 \\
\hline Theor., MeV & 2981 & 3104 & 3528 & 3514 & 3449 & 3552 \\
\hline
\end{tabular}

TABLE V. Masses of the low-lying $S$ - and $P$-wave $\bar{c} c$ mesons.

\begin{tabular}{|c|c|c|c|c|c|c|}
\hline Meson & $\eta_{b}(1 S)$ & $\Upsilon(1 S)$ & $h_{b}(1 P)$ & $\chi_{b_{1}}(1 P)$ & $\chi_{b_{0}}(1 P)$ & $\chi_{b_{2}}(1 P)$ \\
\hline$J^{P}$ & $0^{-+}$ & $1^{--}$ & $1^{+-}$ & $1^{++}$ & $0^{++}$ & $2^{++}$ \\
\hline${ }^{2 S+1} L_{J}$ & ${ }^{1} S_{0}$ & ${ }^{3} S_{1}$ & ${ }^{1} P_{1}$ & ${ }^{3} P_{1}$ & ${ }^{3} P_{0}$ & ${ }^{3} P_{2}$ \\
\hline Exp., MeV $[5]$ & 9398 & 9460 & 9899 & 9893 & 9859 & 9912 \\
\hline Theor., MeV & 9394 & 9459 & 9902 & 9895 & 9871 & 9912 \\
\hline
\end{tabular}

TABLE VI. Masses of the low-lying $S$ - and $P$-wave $\bar{b} b$ mesons.

\begin{tabular}{|c|c|c|c|c|c|c|c|}
\hline Parameter & $\sigma, \mathrm{GeV}^{2}$ & $\alpha_{S}^{\bar{c} q}=\alpha_{S}^{\bar{b} q}=\alpha_{S}^{\bar{c} c}$ & $\alpha_{S}^{\bar{b} b}$ & $C_{0}^{\bar{c} q}, \mathrm{MeV}$ & $C_{0}^{\bar{b} q}, \mathrm{MeV}$ & $C_{0}^{\bar{c} c}, \mathrm{MeV}$ & $C_{0}^{\bar{b} b}, \mathrm{MeV}$ \\
\hline Extracted from fit for & $\bar{c} q$ & $\bar{c} q$ & $\bar{b} b$ & $\bar{c} q$ & $\bar{b} q$ & $\bar{c} c$ & $\bar{b} b$ \\
\hline Listed in Table \# & $\mathrm{I}$ & $\mathrm{I}$ & $\mathrm{VI}$ & $\mathrm{I}$ & II & $\mathrm{V}$ & $\mathrm{VI}$ \\
\hline Value & 0.16 & 0.54 & 0.42 & 330 & 70 & 369 & 50 \\
\hline
\end{tabular}

TABLE VII. Parameters of the model fixed from the fits to the data.

and orbitally $(l=2)$ excited $D$ and $B$ mesons are calculated in the same framework and are confronted with the existing experimental data. The results of calculations and the hypotheses concerning a possible identification of the experimentally observed mesons are contained in Tables III] and IV] If these hypotheses are ranked according to the mean quadratic deviation of the theoretical predictions from the experimental results (evaluated as $\Delta m=\sqrt{\sum_{n=1}^{N}\left(m_{n}^{\text {th }}-m_{n}^{\exp }\right)^{2} / N}$, with $N$ denoting the number of states analysed, and quoted in parentheses for each hypothesis) then hypotheses $\mathcal{D}_{1}$ and $\mathcal{B}_{4}$ should be accepted as the most reliable. The details of the experimental situation with the $B_{J}(5840)$ and $B_{J}(5960)$ candidates can be found in Ref. [7]. Our results are qualitatively compatible with similar predictions previously made for the excited $D$ mesons in Ref. [56] (in the QCD string approach) and with those obtained recently for the excited $D$ and $B$ mesons in Refs. [57, 58, (in the framework of the constituent quark model of Ref. [59]). It should be noticed that the masses of the excited heavylight mesons predicted in the present work in the framework of the QCD string model typically lie somewhat lower than those obtained in Ref. [58] that results in a slightly different suggestion for the identification of the experimentally observed mesons with the theoretically predicted states. The origin of the discrepancy should come from the fact (i) that relativistic dynamics is taken into account in this work while the model used in Ref. [58] is essentially nonrelativistic; (ii) that, contrary to the purely potential approach used in Ref. [58, the proper dynamics of the QCD string is taken into account in our model, which provides an additional negative contribution to the energy - see Eq. (3); and (iii) that the variational einbein field method used in this work may somewhat overestimate the value of the wave function at the origin which governs the mass splitting between the $n^{1} S_{0}$ and $n^{3} S_{1}$ states. A detailed comparison with other approaches and models as well as the relevant references can be found, for example, in Refs. [56, 58,

\section{RESULTS FOR HEAVY-QUARK HYBRIDS}

We now proceed to hybrids. For the states containing the $c$ quark, the string tension and the strong coupling constant are fixed from the spectrum of the heavy-light mesons and the constant $C_{0}^{\bar{c} c}$ is fixed from the spectrum of low-lying $\bar{c} c$ mesons - see Table VII.

From TableV, one can see that the model describes the experimental spectrum of the $\bar{c} c$ states with high accuracy, which is especially remarkable given that only the 
overall shift of the spectrum $C_{0}^{\bar{c} c}$ was adjusted, and all other parameters were fixed earlier. Similarly, the spectrum of the low-lying $\bar{b} b$ mesons is also described with the same string tension while, in agreement with the discussion above, the strong coupling constant is somewhat decreased in this case; notice that its fitted value complies well with the estimate from Eq. (14). The obtained values of the $\alpha_{S}^{\bar{b} b}$ and $C_{0}^{\bar{b} b}$ are quoted in Table VII Similarly to the $\bar{c} c$ states, the spectrum of the low-lying $\bar{b} b$ mesons is remarkably well described by the model-see Table VI

To search for the eigenenergies of the Hamiltonian (2) we employ the variational technique described in detail in Ref. 36. In particular, we use the Harmonic Oscillator trial wave function $\left[\exp \left(-\beta^{2} \mu r^{2} / 2\right)\right.$ multiplied by the appropriate spherical harmonic and Laguerre polynomial], that gives

$$
\begin{aligned}
& \mu(\bar{c} c ; 1 S)=1893 \mathrm{MeV}, \quad \beta^{2}(\bar{c} c ; 1 S)=280 \mathrm{MeV} \\
& \mu(\bar{c} c ; 1 P)=1866 \mathrm{MeV}, \quad \beta^{2}(\bar{c} c ; 1 P)=148 \mathrm{MeV} \\
& \mu(\bar{b} b ; 1 S)=5019 \mathrm{MeV}, \quad \beta^{2}(\bar{b} b ; 1 S)=312 \mathrm{MeV} \\
& \mu(\bar{b} b ; 1 P)=4942 \mathrm{MeV}, \quad \beta^{2}(\bar{b} b ; 1 P)=128 \mathrm{MeV}
\end{aligned}
$$

where, as before, the quark contents of the quarkantiquark system and its quantum numbers are quoted in parentheses.

With the set of the parameters from Table VII we are now in a position to predict the masses of the lowest magnetic $\bar{c} c g$ hybrids. We use the trial wave function $\rho Y_{1 m}(\hat{\rho}) \exp \left(-\beta^{2} M R^{2} / 2\right)$, where $\rho$ is the Jakobi coordinate of the gluon relative to the centre of mass of the quark-antiquark subsystem, $R$ is the standard hyperspherical radius defined for the three-body system $\bar{Q} Q g$, and $M=2 \mu+\mu_{g}$ - see Ref. 36] for further details. Then, the parameters $\mu, \mu_{g}$, and $\beta^{2}$ take the following values (in $\mathrm{MeV}$ ):

$$
\mu(\bar{c} c g)=1778, \quad \mu_{g}(\bar{c} c g)=1104, \quad \beta^{2}(\bar{c} c g)=380 .
$$

The results given in Table VIII can be regarded as an update of the predictions contained in Ref. 36]. They comply well with the predictions found in the literature and obtained in the framework of different approaches. In particular, the bag model predicts the mass of the lowest charm hybrid around $4 \mathrm{GeV}[24,25$. In the flux tube model the low-lying hybrids reside in the region around 4.1-4.2 GeV 60]. Adiabatic approximation for heavy quarks in the QCD string model in the formalism of auxiliary fields also gives a similar result, namely $4.2 \pm 0.2$ $\mathrm{GeV}$ for the hybrid with the exotic quantum numbers $1^{-+} 61$. The mass of the tensor hybrid is predicted to be $4.12 \mathrm{GeV}$ in the potential quark model [30]. Various lattice calculations also place charmonium hybrids at around $4.4 \mathrm{GeV}$ [9, 19, 20.

Analogously, the parameters from Table VII allow one to predict the masses of the bottomonium hybrids which are collected in Table IX. The corresponding values of the parameters $\mu, \mu_{g}$, and $\beta^{2}$ are (in $\mathrm{MeV}$ )

$$
\mu(\bar{b} b g)=4813, \quad \mu_{g}(\bar{b} b g)=1194, \quad \beta^{2}(\bar{b} b g)=330 .
$$

It is important to notice that the vector hybrid is expected to have the mass around $11 \mathrm{GeV}$, that is it resides in the vicinity of the $\Upsilon(11020)$ resonance. This result complies well with the predictions from the lattice which place the bottomonium hybrid at 10900(100) MeV [18].

\section{DISCUSSION AND CONCLUSIONS}

In this paper, we revised the QCD string approach in application to heavy-light mesons and hybrids containing heavy quarks. In contrast to earlier works, the number of parameters is minimised and the same set of parameters, consistent with phenomenology, is used to describe simultaneously masses of the radially and orbitally excited $D$ and $B$ mesons, low-lying $\bar{c} c$ and $\bar{b} b S$-wave and $P$-wave mesons, and the lowest magnetic $\bar{c} c g$ and $\bar{b} b g$ hybrids. The approach used in this work, on one hand being rather simple and physically transparent, on the other hand demonstrates a high accuracy, and thus its predictions for yet not observed or not confirmed states can be regarded as rather reliable. In particular, the $B_{1}(5721), B_{J}^{*}(5732)$, and $B_{2}^{*}(5747)$ mesons are identified as the axial vector $\left(1^{+}\right)$, the scalar $\left(0^{+}\right)$, and the tensor $\left(2^{+}\right)$members of the $P$-level $J^{+}(J=0,1,2)$ quadruplet, respectively. The last remaining member of the same quadruplet is predicted to be broad and to possess the mass around $5713 \mathrm{MeV}$. Also, the states $B_{J}(5840)$ and $B_{J}(5960)$ reported recently by the $\mathrm{LHCb}$ Collaboration are most probably the $2^{1} S_{0}$ and the $1 D_{2}^{h}$ (here $h$ stands for the heavy member of the $1 D_{2}$ doublet), respectively. Finally, in the same scheme, the CDF meson B(5970) can be identified with the $1^{3} D_{3}$ state (such an identification was also suggested in Ref. 62]). Meanwhile, we agree with the conclusion of Ref. [58] that other hypotheses for these states should be considered seriously too, and that additional important, probably decisive, information should be provided by the data on the decay modes of the states under study.

Finally, the masses of the lowest magnetic charmonium and bottomonium hybrids are calculated in the same model and with the parameters previously fixed from the spectrum of ordinary mesons. Interestingly, the vector bottomonium hybrid is predicted to have the mass of approximately $11.04 \mathrm{GeV}$ that is very close to the mass of the $\Upsilon(11020)$ resonance. This may imply a considerable admixture of the hybrid component in its wave function, in addition to the $\bar{b} b$ component which can be identified with the radially excited $\Upsilon(6 S)$ genuine $\bar{b} b$ quarkonium.

Identification of the positive-parity $B_{J}$ mesons given in Table II, together with the well-established masses of the pseudoscalar $B$ meson and the vector $B^{*}$ meson [5],

$$
m_{B}=5279 \mathrm{MeV}, \quad m_{B^{*}}=5325 \mathrm{MeV},
$$

allows one to estimate the positions of the lowest open- 


\begin{tabular}{|c|l|l|l|l|}
\hline$J^{P}$ & $0^{-+}$ & $1^{-+}$ & $1^{--}$ & $2^{-+}$ \\
\hline Theor., MeV & 4296 & 4358 & 4430 & 4484 \\
\hline
\end{tabular}

TABLE VIII. Predictions for the masses of the lowest hybrids $c \bar{c} g$.

\begin{tabular}{|c|c|c|c|c|}
\hline$J^{P}$ & $0^{-+}$ & $1^{-+}$ & $1^{--}$ & $2^{-+}$ \\
\hline Theor., MeV & 10990 & 11013 & 11038 & 11057 \\
\hline
\end{tabular}

TABLE IX. Predictions for the masses of the lowest hybrids $b \bar{b} g$.

bottom thresholds with the $B_{J}$ family mesons involved,

$$
\begin{gathered}
M\left(B \bar{B}_{1}(5721)\right)=11005 \mathrm{MeV}, \\
M\left(B^{*} \bar{B}_{1}(5721)\right)=11050 \mathrm{MeV}, \\
M\left(B^{*} \bar{B}_{2}^{*}(5747)\right)=11064 \mathrm{MeV},
\end{gathered}
$$

where only the narrow $B_{J}$ mesons are taken into account since the experimental observation of their broad partners in the open-bottom final states of the form 21) does not look feasible. The thresholds which involve two $B_{J}$ mesons lie considerably higher, at around $11.5 \mathrm{GeV}$. Therefore, while the production channels for the $B_{J}$ family are kinematically closed for the $B$-factories working at the energies of the $\Upsilon(4 S)$ and $\Upsilon(10860)$ vector resonances, they could be observed at Belle-II in the decays of $\Upsilon(11020)$. This possibility requires an additional study though. Since the broad members of the positive-parity quadruplet not considered here originate from the $P_{1 / 2}$ heavy-quark state, we concentrate on the $P_{3 / 2}$ term. It has to be noticed then that production of a heavy-light meson from the $P_{3 / 2}$ state accompanied by a $S$-wave $B^{(*)}$ meson-see Eq. 21 - is only possible if the produced light-quark pair has the total momentum equal to 1. This condition is not fulfilled for the vector bottomonium where $j_{q \bar{q}}=0$, and therefore the amplitude for its decay into the $B^{(*)} B\left(P_{3 / 2}\right)$ pair is suppressed in the heavy-quark limit [39], which is certainly a good approximation for the $b$ quark. Meanwhile, open-flavour decays of a $\bar{b} b g$ hybrid proceed through the gluon conversion into a light quark-antiquark pair which therefore carries the quantum numbers of the vector, in particular, $j_{q \bar{q}}=1$. This implies that there is no suppression for the amplitude of the vector hybrid decay into a pair of one $S$-wave and one $P$-wave open-bottom meson - see Ref. 36, 52 for the corresponding recoupling coefficients. Therefore, the decays to the final states from Eq. (21) [especially to the first one, with the threshold located below the nominal $\Upsilon(11020)$ mass] could be used as test modes for the bottomonium hybrid in the vicinity of $11 \mathrm{GeV}$. It should be noticed, however, that this conclusion is valid only in the

\footnotetext{
1 Although the last two thresholds in Eq. 21 formally lie above the nominal mass of the $\Upsilon(11020)$ resonance, due to the finite
}

strict heavy-quark limit $m_{b} \rightarrow \infty$. For a finite $m_{b}$, corrections of two types have to be taken into account. On one hand, there exist corrections to the heavy-quark spin symmetry limit which are controlled by the small parameter $\Lambda_{\mathrm{QCD}} / m_{b}$ and which are expected to be quite small, too-indeed, constraints from the heavy-quark spin symmetry are typically very well met in bottomonium systems. On the other hand, as was mentioned above, the physical meson $B_{1}(5721)$ is a mixture of both $B_{1 / 2}$ and $B_{3 / 2}$ states governed by the mixing angle $\theta_{B}$ - see Fig. 1 and Table $\mathrm{VI}$. Therefore, the probability of the decay $\Upsilon(11020) \rightarrow B \bar{B}_{1}(5721)$ is proportional to $\sin ^{2} \theta_{B}$ for the $\Upsilon(11020)$ as a genuine $\bar{b} b$ quarkonium, and it is proportional to $\cos ^{2} \theta_{B}$ for the hybrid. For $\theta_{B} \ll 1$, this mode could have been regarded as a smoking gun for the hybrid nature of the $\Upsilon(11020)$ resonance. Meanwhile, the actual mixing angle is $\theta_{B} \approx 24^{\circ}$ that gives $\sin ^{2} \theta_{B} \approx 0.17$ and $\cos ^{2} \theta_{B} \approx 0.83$. Thus, although $\sin ^{2} \theta_{B} \ll \cos ^{2} \theta_{B}$, it remains to be seen whether or not such a suppression factor is sufficient to allow one to distinguish between the genuine quarkonium and the hybrid lying at around $11 \mathrm{GeV}$. However, in any case, studies of the decays to the final states from Eq. 21 ${ }^{1}$ appear to be a very interesting and promising source of information for the phenomenology of bottomonium, and therefore data taking at $B$-factories of the next generation at the energies around $11 \mathrm{GeV}$ and above look quite promising (see also the discussions in Ref. [2]). Given that the above decays are expected to occur near their respective thresholds, the corresponding line shapes should demonstrate a typical threshold behaviour that makes them appealing also for various studies of the threshold phenomena.

\section{ACKNOWLEDGMENTS}

The authors would like to thank F.-K. Guo and M. Voloshin for discussions. This work is supported by the Russian Science Foundation (Grant No. 15-12-30014).

width of the latter as well as the finite widths of the $B$ mesons, they might be possible to observe. 
[1] N. Brambilla et al., Eur. Phys. J. C 71, 1534 (2011).

[2] A. G. Drutskoy, F. K. Guo, F. J. Llanes-Estrada, A. V. Nefediev, and J. M. Torres-Rincon, Eur. Phys. J. A 49, 7 (2013).

[3] N. Brambilla et al., Eur. Phys. J. C 74, 2981 (2014).

[4] A. Bondar et al. (Belle Collaboration), Phys. Rev. Lett. 108, 122001 (2012).

[5] K. A. Olive et al. (Particle Data Group Collaboration), Chin. Phys. C 38, 090001 (2014).

[6] T. A. Aaltonen et al. (CDF Collaboration), Phys. Rev. D 90, 012013 (2014).

[7] R. Aaij et al. (LHCb Collaboration), JHEP 1504, 024 (2015)

[8] B. Aubert et al. (BaBar Collaboration), Phys. Rev. Lett. 95, 142001 (2005).

[9] X. Q. Luo and Y. Liu, Phys. Rev. D 74, 034502 (2006); Erratum: Phys. Rev. D 74, 039902 (2006).

[10] A. Le Yaouanc, L. Oliver, O. Pene, J. C. Raynal, and S. Ono, Z. Phys. C 28, 309 (1985).

[11] F. Iddir, S. Safir, and O. Pene, Phys. Lett. B 433, 125 (1998).

[12] Yu. S. Kalashnikova, Z. Phys. C 62, 323 (1994).

[13] E. Kou and O. Pene, Phys. Lett. B 631, 164 (2005).

[14] N. Isgur, R. Kokoski, and J. Paton, Phys. Rev. Lett. 54, 869 (1985).

[15] F. E. Close and P. R. Page, Nucl. Phys. B 443, 233 (1995).

[16] G. Pakhlova et al. (Belle Collaboration), Phys. Rev. D 80, 091101 (2009).

[17] Q. Wang, M. Cleven, F. K. Guo, C. Hanhart, U.G. Meißner, X. G. Wu, and Q. Zhao, Phys. Rev. D 89, 034001 (2014).

[18] K. J. Juge, J. Kuti, and C. J. Morningstar, Phys. Rev. Lett. 82, 4400 (1999).

[19] C. Michael, arXiv:hep-ph/0308293.

[20] Y. Liu and X. Q. Luo, Phys. Rev. D 73, 054510 (2006).

[21] T. Burch and C. Ehmann, Nucl. Phys. A 797, 33 (2007).

[22] J. J. Dudek, R. G. Edwards, N. Mathur, and D. G. Richards, Phys. Rev. D 77, 034501 (2008).

[23] J. J. Dudek and E. Rrapaj, Phys. Rev. D 78, 094504 (2008).

[24] M. S. Chanowitz and S. R. Sharpe, Nucl. Phys. B 222, 211 (1983); Erratum: Nucl. Phys. B 228, 588 (1983).

[25] T. Barnes, F. E. Close, F. de Viron, and J. Weyers, Nucl. Phys. B 224, 241 (1983).

[26] T. Barnes, F. E. Close, and E. S. Swanson, Phys. Rev. D 52, 5242 (1995).

[27] S. R. Cotanch and F. J. Llanes-Estrada, Nucl. Phys. A 689, 481 (2001).

[28] I. J. General, S. R. Cotanch, and F. J. Llanes-Estrada, Eur. Phys. J. C 51, 347 (2007).

[29] F. J. Llanes-Estrada and S. R. Cotanch, Phys. Lett. B 504, 15 (2001).

[30] E. Abreu and P. Bicudo, J. Phys. G 34, 195 (2007).

[31] D. Horn and J. Mandula, Phys. Rev. D 17, 898 (1978).

[32] Yu. A. Simonov, Phys. At. Nucl. 64, 1876 (2001) [Yad. Fiz. 64, 1959 (2001)].

[33] Yu. S. Kalashnikova and Yu. B. Yufryakov, Phys. Lett. B 359, 175 (1995).

[34] Yu. A. Simonov, Phys. At. Nucl. 68, 1294 (2005) [Yad. Fiz. 68, 1347 (2005)].
[35] Yu. S. Kalashnikova and D. S. Kuzmenko, Phys. At. Nucl. 66, 955 (2003) [Yad. Fiz. 66, 988 (2003)].

[36] Yu. S. Kalashnikova and A. V. Nefediev, Phys. Rev. D 77, 054025 (2008).

[37] F. Buisseret and C. Semay, Phys. Rev. D 74, 114018 (2006).

[38] F. Buisseret, V. Mathieu, C. Semay and B. SilvestreBrac, Eur. Phys. J. A 32, 123 (2007).

[39] X. Li and M. B. Voloshin, Phys. Rev. D 88, 034012 (2013).

[40] A. Di Giacomo, H. G. Dosch, V. I. Shevchenko, and Yu. A. Simonov, Phys. Rep. 372, 319 (2002).

[41] A. Yu. Dubin, A. B. Kaidalov, and Yu. A. Simonov, Phys. Lett. B 323, 41 (1994).

[42] A. Yu. Dubin, A. B. Kaidalov, and Yu. A. Simonov, Phys. Lett. B 343, 310 (1995).

[43] L. Brink, P. Di Vecchia, and P. S. Howe, Nucl. Phys. B 118, 76 (1977).

[44] Yu. S. Kalashnikova and A. V. Nefediev, Phys. At. Nucl. 60, 1389 (1997) [Yad. Fiz. 60, 1529 (1997)].

[45] Yu. A. Simonov, Nuovo Cimento A 107, 2629 (1994).

[46] A. B. Kaidalov, and Yu. A. Simonov, Phys. Lett. B 477, 163 (2000).

[47] Yu. S. Kalashnikova, A. V. Nefediev, and Yu. A. Simonov, Phys. Rev. D 64, 014037 (2001).

[48] Yu. A. Simonov, in Proceedings of the XVII International School of Physics "QCD: Perturbative or Nonperturbative," Lisbon, 1999, edited by L. S. Ferreira, P. Nogueira, and J. I. Silva-Marcos (World Scientific, Singapore, 2000), p. 60.

[49] Yu. S. Kalashnikova and A. V. Nefediev, Phys. Lett. B 492, 91 (2000).

[50] Yu. S. Kalashnikova and A. V. Nefediev, Phys. Lett. B 530, 117 (2002).

[51] Yu. S. Kalashnikova and A. V. Nefedev, Phys. At. Nucl. 68, 650 (2005) [Yad. Fiz. 68, 681 (2005)].

[52] Yu. S. Kalashnikova and A. V. Nefediev, Phys. At. Nucl. 72, 333 (2009).

[53] Yu. A. Simonov, Phys. At. Nucl. 74, 1223 (2011).

[54] Yu. A. Simonov, Phys. Lett. B 515, 137 (2001).

[55] A. B. Kaidalov and Yu. A. Simonov, Phys. Lett. B 477, 163 (2000).

[56] A. M. Badalian and B. L. G. Bakker, Phys. Rev. D 84, 034006 (2011).

[57] D. M. Li, P. F. Ji, and B. Ma, Eur. Phys. J. C 71, 1582 (2011).

[58] Q. F. Lü, T. T. Pan, Y. Y. Wang, E. Wang and D. M. Li, Phys. Rev. D 94, no. 7, 074012 (2016).

[59] O. Lakhina and E. S. Swanson, Phys. Lett. B 650, 159 (2007)

[60] N. Isgur and J. E. Paton, Phys. Rev. D 31, 2910 (1985).

[61] F. Buisseret and V. Mathieu, Eur. Phys. J. A 29, 343 (2006).

[62] L. Y. Xiao and X. H. Zhong, Phys. Rev. D 90, 074029 (2014). 


\section{Appendix A: Splitting scheme for $P$-level heavy-light mesons}

For an arbitrary heavy-quark mass $m_{Q}$ the physical observed states with the quantum numbers $J^{P}=1^{+}$, conveniently denoted as $P_{1}^{l}$ and $P_{1}^{h}$ for the light and the heavy member of the doublet, respectively, are presented as particular combinations of the $\left\{{ }^{2 S+1} L_{J}\right\}$ basis vectors ${ }^{1} P_{1}$ and ${ }^{3} P_{1}$,

$$
\left(\begin{array}{c}
P_{1}^{l} \\
P_{1}^{h}
\end{array}\right)=\left(\begin{array}{cc}
\cos \theta\left(m_{Q}\right) & -\sin \theta\left(m_{Q}\right) \\
\sin \theta\left(m_{Q}\right) & \cos \theta\left(m_{Q}\right)
\end{array}\right)\left(\begin{array}{c}
{ }^{1} P_{1} \\
{ }^{3} P_{1}
\end{array}\right)
$$

The mixing matrix in Eq. A1 can be found as

$$
\left(\begin{array}{c}
\frac{E_{2}^{(0)}-E_{1}}{\sqrt{\left(E_{2}^{(0)}-E_{1}\right)^{2}+V^{2}}}-\frac{V}{\sqrt{\left(E_{2}^{(0)}-E_{1}\right)^{2}+V^{2}}} \\
\frac{E_{2}^{(0)}-E_{2}}{\sqrt{\left(E_{2}^{(0)}-E_{2}\right)^{2}+V^{2}}}-\frac{V}{\sqrt{\left(E_{2}^{(0)}-E_{2}\right)^{2}+V^{2}}}
\end{array}\right)
$$

where

$$
E_{1}^{(0)} \equiv\left\langle{ }^{1} P_{1}|H|^{1} P_{1}\right\rangle, \quad E_{2}^{(0)} \equiv\left\langle{ }^{3} P_{1}|H|^{3} P_{1}\right\rangle
$$

$$
V \equiv\left\langle{ }^{1} P_{1}|H|^{3} P_{1}\right\rangle=\left\langle{ }^{3} P_{1}|H|^{1} P_{1}\right\rangle,
$$

and $E_{1}$ and $E_{2}$ are the solutions of the secular equation,

$$
\operatorname{det}\left(\begin{array}{cc}
E_{1}^{(0)}-E & V \\
V & E_{2}^{(0)}-E
\end{array}\right)=0
$$

that is

$$
E_{1,2}=\frac{1}{2}\left(E_{2}^{(0)}-E_{1}^{(0)}\right) \pm \sqrt{\frac{1}{4}\left(E_{2}^{(0)}-E_{1}^{(0)}\right)^{2}+V^{2}}
$$

In the strict heavy-quark limit, $m_{Q} \rightarrow \infty$, the mixing matrix from Eq. A1 takes a universal form which corresponds to the "ideal" mixing,

$$
\left(\begin{array}{l}
P_{1}^{l(0)} \\
P_{1}^{h(0)}
\end{array}\right)=\left(\begin{array}{cc}
\cos \theta(\infty) & -\sin \theta(\infty) \\
\sin \theta(\infty) & \cos \theta(\infty)
\end{array}\right)\left(\begin{array}{c}
{ }^{1} P_{1} \\
{ }^{3} P_{1}
\end{array}\right),
$$

where $\cos \theta(\infty)=1 / \sqrt{3}$ and $\sin \theta(\infty)=\sqrt{2 / 3}$.

Obviously, in the same heavy-quark limit, one can identify the physical states $P_{1}^{l(0)}$ and $P_{1}^{h(0)}$ with the states $P_{1 / 2}$ and $P_{3 / 2}$ in heavy-quark limit, respectively ${ }^{2}$ Then Eqs. A1 and A7) together give relation (16) between the wave functions of the physical states and the heavy-quark basis states, with $\theta=\theta\left(m_{Q}\right)-\theta(\infty)$. Dependence of the angle $\theta$ from the heavy-quark mass is depicted in Fig. 1 .

\footnotetext{
2 The inversed level ordering, that is $M\left(P_{1 / 2}\right)>M\left(P_{3 / 2}\right)$, is also possible in the given model - see Ref. 50 - however, the updated
} 